\title{
Outline of an experimental design aimed to detect protein A mirror image in solution
}

\author{
Osvaldo A Martin ${ }^{\text {Corresp., } 1}$, Yury Vorobjev ${ }^{2}$, Harold A Scheraga ${ }^{3}$, Jorge A Vila ${ }^{1,3}$ \\ 1 Instituto de Matemática Aplicada San Luis, UNSL-CONICET, San Luis, Argentina \\ 2 Institute of Chemical Biology and Fundamental Medicine, Siberian Branch of the Russian Academy of Science, Novosibirsk, Russia \\ 3 Baker Laboratory of Chemistry and Chemical Biology, Cornell University, Ithaca, New York, United States \\ Corresponding Author: Osvaldo A Martin \\ Email address: omarti@unsl.edu.ar
}

There is abundant theoretical evidence indicating that a mirror image of Protein A may occur during the protein folding process. However, as to whether such mirror image exists in solution is an unsolved issue. Here we provide outline of an experimental design aimed to detect the mirror image of Protein $A$ in solution. The proposal is based on computational simulations indicating that the use of a mutant of protein $\mathrm{A}$, namely $\mathrm{Q} 10 \mathrm{H}$, could be used to detect the mirror image conformation in solution. Our results indicate that the native conformation of the protein A should have a pKa, for the Q10H mutant, at $\approx 6.2$, while the mirror-image conformation should have a pKa close to $\approx 7.3$. Naturally, if all the population is in the native state for the $\mathrm{Q} 10 \mathrm{H}$ mutant, the pKa should be $\approx 6.2$, while, if all are in the mirror-image state, it would be $\approx 7.3$, and, if it is a mixture, the pKa should be larger than 6.2 , presumably in proportion to the mirror population. In addition, evidence is provided indicating the tautomeric distribution of $\mathrm{H} 10$ must also change between the native and mirror conformations. Although this may not be completely relevant for the purpose of determining whether the protein A mirror image exists in solution, it could provide valuable information to validate the pKa findings. We hope this proposal will foster experimental work on this problem either by direct application of our proposed experimental design or serving as inspiration and motivation for other experiments. 


\section{Outline of an experimental design aimed to detect a protein A mirror image in solution}

3

\author{
Osvaldo A. Martin ${ }^{1}$, Yury Vorobjev ${ }^{2}$, Harold A. Scheraga ${ }^{3}$, and Jorge A. \\ Vila ${ }^{1,3}$
}

\author{
${ }^{1}$ Instituto de Matemática Aplicada San Luis, UNSL-CONICET, San Luis, Argentina \\ ${ }^{2}$ Institute of Chemical Biology and Fundamental Medicine, Siberian Branch of the \\ Russian Academy of Science, Novosibirsk, Russia \\ ${ }^{3}$ Baker Laboratory of Chemistry and Chemical Biology, Cornell University, Ithaca, New \\ York, United States
}

Corresponding author:

Osvaldo A. Martin ${ }^{1}$

Email address: omarti@unsl.edu.ar

\section{INTRODUCTION}

A mirror image conformation is one that looks approximately like the specular image of the native state. We say approximately because we do not require the amino acids to be specular images, but only the overall topology of the molecule. At least for some proteins, the mirror image will be energetically very close to the native state and thus it could also exist in solution. Among these proteins, we will focus our attention on the B-domain of staphylococcal protein A [PDB ID: 1BDD; a three-helix bundle] Gouda et al. (1992). This protein has been the subject of extensive theoretical Olszewski et al. (1996); Vila et al. (2003); Garcia and Onuchic (2003); Lee et al. (2006); Kachlishvili et al. (2014) and experimental Deisenhofer (1981); Gouda et al. (1992); Bai et al. (1997); Myers and Oas (2001); Sato et al. (2004); Dimitriadis et al. (2004); Noel et al. (2012) studies because of its biological importance and small size. In contrast to this, the mirror-image conformation has been subject of limited discussion Olszewski et al. (1996); Vila et al. (2003); Garcia and Onuchic (2003); Noel et al. (2012); Kachlishvili et al. (2014). The reason for this might be that the mirror image conformation of this protein has been observed only in some theoretical studies with different force fields but it has never been detected experimentally. As to whether this conformation is an artifact of the simulations or is difficult to observe the conformation experimentally, remains to be solved.

Difficulties for experiments to detect the mirror-image conformation arise precisely because the secondary structures of the mirror-image and the native conformation of protein A are identical and the 
structural difference between these conformations are subtle Kachlishvili et al. (2014). Because of this, use of simple experiments such as circular dichroism, used to estimate the fraction of secondary-structure content, or more sophisticative technique, such as nuclear magnetic resonance (NMR) spectroscopy, e.g., to monitor the ${ }^{13} C$ chemical shift changes that may occur at residue-level Kachlishvili et al. (2014), are useless for an accurate characterization of the mirror image conformation. A strong motivation to propose alternative methods to explore the possible coexistence in solution of the native and mirror-image conformation of protein A, comes from older evidence indicating that the mirror-image conformation could be a possible solution to the NMR-determined structure of protein A Gouda et al. (1992). Indeed, according to Gouda et al. Gouda et al. (1992), “... distance-geometry calculations resulted in 41 solutions, which had correct polypeptide folds excluding 14 mirror-image substructures..." However, the mirrorimage structures were excluded from the analysis of Gouda et al. Gouda et al. (1992) without providing any reason. It seems that the decision was adopted because the "mirror-image" satisfies the NOE constraints but contain D-amino acid residues (personal communication with Ichio Shimada).

Overall, we propose here a proof-of-concept of an experimental design aimed to solve this problem. Initially we will show, by using ROSETTA, Bradley et al. (2005) that a mutant of protein A, hereafter the Q10H protein, exhibits the ability to fold into the native conformation (see Figure 1) as well as into the mirror-image conformation (see Figure 2). Later, we estimate the fraction of the native and mirror-image populations of the protein $\mathrm{Q} 10 \mathrm{H}$ by using a recently introduced method, that take into account the protein dynamics in water by using a constant-pH MD simulation, to accurately determine the pKa values of ionizable residues, and fractions of ionized and tautomeric forms of histidine (His), in proteins at a given fixed $\mathrm{pH}$ Vorobjev et al. (2018). Indeed, we explore the dependence of the electrostatically-calculated $\mathrm{pKa}$ and fractions of the imidazole ring forms of $\mathrm{H} 10$ as a function of $\mathrm{pH}$ for both the native-like and mirror-image conformations.

\section{MATERIALS AND METHODS}

In this section we will give a brief reference to existent theoretical methods aimed to predict $(i)$ the 3D structure of proteins accurately; Bradley et al. (2003) or determine (ii) the pKa values of ionizable residues and fractions of ionized and tautomeric forms of histidine (His) and acid residues in proteins, at a given fixed $\mathrm{pH}$ Vorobjev et al. $(2008,2018)$.

\section{Determination of the native and image-mirror conformations of protein Q10H}

To generate the native and mirror-image conformations of protein A Q10H we used the fast-relax protocol from Rosetta, Chaudhury et al. (2010); Bradley et al. (2005) this is an all-atom refinement protocol consisting of several rounds of repacking and energy minimization. The repulsive part of the Van der Waals energy function is annealed from $2 \%$ to $100 \%$. Essentially the algorithm explores the local conformational space around the starting structure with a radius of 2 to $3 \AA$ of rmsd (for the $C^{\alpha}$ ). We performed several rounds of fast-relax using the following genetic-like algorithm:

1. For a given conformation of protein A mutate it by replacing Q10 with $\mathrm{H} 10$

2. Use the mutant as the starting point of 200 independent rounds of the fast-relaxation protocol

3. Choose 10 conformations; 2 at random and the 8 lowest-energy conformations

4. For each one of those conformations use fast-relaxation to generate 100 independent rounds (for a total of 1000 conformations)

5. repeat, from step 3,40 times

6. keep the lowest energy conformation from all the rounds

We started from 2 different conformations. For the native conformation we used 1BDD Gouda et al. (1992). For the mirror image we started from a mirror-image conformation previously obtained by Vila et al, Vila et al. (2003).

The Rosetta energy score of the lowest energy conformations for the native and image-mirror of protein Q10H was on par. 


\section{Computation of the pKa and the tautomeric fractions of the imidazole ring of $\mathrm{H} 10$}

The native-like and mirror-image conformations of protein Q10H, generated as describe in the previous section, were used as input files for the calculations of the pKa of all ionizable residues in the sequence as well as the fractions of the ionized $H^{+}$and the tautomeric $N^{\varepsilon 2}-H$ and $N^{\delta 1}-H$ forms of the imidazole ring of H10. In particular, as it is well known, the tautomeric determination of the imidazole ring of His is both a very important problem in structural biology Schnell and Chou (2008); Bermúdez et al. (2014) and a challenging task Machuqueiro and Baptista (2011). For this reason, a recently introduced electrostatic-based method to determine the pKa values of ionizable residues and fractions of ionized and tautomeric forms of histidine (His) and acid residues in proteins Vorobjev et al. (2018), is applied here to the analysis of protein A mutant Q10H. Protein dynamics in water, at a given $\mathrm{pH}=7.0$, was taken into account by constant-pH MD simulation Vorobjev et al. $(2017,2018)$ of both the native and mirror-image conformations of the Q10H mutant.

Protein dynamics in water was modeled by MD simulations with implicit solvent, namely using the Lazaridis-Karplus solvent model Lazaridis and Karplus (1999) with the BioPASED program Popov and Vorob'ev (2010). For the MD simulation, the following three-step protocol was used. First step, determination of an equilibrium protein structure at temperature $300 \mathrm{~K}$ and $\mathrm{pH} 7.0$ using the next three step procedure: (i) building a full atomic protein structure, i.e. with all hydrogen atoms added; this means, for example, that each His residue needs to be built up in the most probable form, i.e. in the ionized $\mathrm{H}^{+}$ form or in the most probable neutral tautomer, $N^{\delta 1}-H$ (HD1 or HID) and $N^{\varepsilon 2}-H$ (HE2 or HIE); (ii) the crystal structure with all the assigned hydrogen atoms and histidine forms was energy optimized in implicit solvent using a conjugate gradient method; (iii) the system is heated slowly from 1 to $300 \mathrm{~K}$ during $250 \mathrm{ps}$; and (iv) a final equilibration at $300 \mathrm{~K}$, during 0.5-1 ns, was carried out. Step 2: generation of a representative set of 3D protein structures as a collections of snapshots each 50 ps along equilibrium MD trajectory during $25 \mathrm{~ns}$ snapshots taken every $50 \mathrm{ps}$ time-interval. Step 3: for each snapshot, the pKa's of all ionizable residues is computed, as well as the fractions of two neutral tautomers of His and the acid residues, by carrying out an MC calculation with GB-MSR6c as an implicit solvent model. Finally, an average $\mathrm{pKa}$ 's for each ionizable residue as well as the fraction of ionized and two tautomers of histidine and neutral form of acid residues of the protein are calculated.

The ionization constants $\mathrm{pKa}$ and the fractions of ionized and two neutral tautomers of histidine at constant pH 7.0 are modeled by MD simulations at constant pH Vorobjev et al. (2017, 2018). During the pH-constant MD simulations all acid (Asp, Glu) and base (Lys, Arg) residues were kept in the ionized state because their respective pKo's (3.5, 4.0, and 10.5, 12.5, respectively) are shifted by more than 2.5 $\mathrm{pK}$ units from the $\mathrm{pH}$ (7.0) at which the calculations were carried out (see Table S1 in supplemental files). On the other hand, the two existent histidine residues, namely $\mathrm{H} 10$ and $\mathrm{H} 19$, were considered to be electrostatically couple residues having nine ionization states, namely, 00, 01, 02, 10, 11, 12, 20, 21,22 , where $0,1,2$ represents the ionized and two neutral tautomer states respectively (see Table $\mathrm{S} 2$ in supplemental files). The average potential energy values and it's thermal fluctuations due to molecular dynamics in solvent are estimated along 25ns MD equilibrium trajectory for each of the nine ionization states (see Table S2 in supplemental files). Low energy states, which have occupation number large than 0.01, for histidine residues H10 and H19 along 25ns constant-pH MD trajectory, are shown in Table S3 (see supplemental files). Energy fluctuations of the Q10H protein in solvent along $25 \mathrm{~ns}$ MD trajectory for native-like and mirror-image structures are shown in Figure S1 (see supplemental files). It can be seen, from this figure, that fluctuation of the native-like and mirror image structures are overlapping, i.e. spontaneous transition between native-like and mirror-image structures can occur. The average range of fluctuations of the atomic positions, i.e. in terms of the RMSD, observed along the MD trajectories were 1.4 and $1.3 \AA$ for the native-like and mirror-image structures, respectively. Variation of pKa constant along MD trajectory is presented on Figure S2 (see supplemental files). It can be seen that pKa shift for histidine His 10 are -0.3 and $+0.8 \mathrm{pK}$ units for the native-like and mirror-image protein structures. Such relatively large pKa shift for relatively small proteins can serves as a mark of native-like and mirror-image structures. Occupations of ionization states of His 10 residue versus MD time are shown in Figure S3a and Figure S3b (see supplemental files) for native-like and mirror-mage structures, respectively. It should be noticed, that occupation of different ionization states of His 10 show a large variation, i.e. RMSD from it's average values.

One challenge question is how meaningful the $\mathrm{pK}$ difference computed with our method are. In this regards, we would like to mention that the accuracy of the $\mathrm{pK}$ calculations have been carefully analyzed 
through a series of applications. Indeed, a comparison with experimental data show the method is accurate enough, in terms of a NMR-based methodology, to predict the $\mathrm{pK}$ and tautomeric fractions of six histidine forms on the enzime DFPase from Loligo vulgaris, a 314-residues all- $\beta$ protein containing 94 ionizable residues Vorobjev et al. (2018). In addition, a large test on 297 ionized residues from 34 proteins show that a $57 \%, 86 \%$ and $95 \%$ of the $\mathrm{pK}$ prediction are with an accuracy better than $0.5,1.0$ and $1.5 \mathrm{pK}$ unit respectively Vorobjev et al. (2017). Such range of accuracy is comparable or better than state of the art predictive methods such as the electrostatic-based MCCI2 method Song et al. (2009).

Moreover, the $\mathrm{H} 10 \mathrm{pKa}$ differences between the native-like and the mirror-image conformations of Q10H protein does not disappear but kept constant $(\approx 1.1 \mathrm{pK}$ units) between 9 ns- $25 \mathrm{~ns}$ of the $\mathrm{pH}$-constant MD simulation (see Figure S2 and table S3 in supplemental files), hence, given further confidence on the accuracy of the $\mathrm{pK}$ shift predictions.

Since the error of our pKa predictive method can be positive or negative, there is a non-negligible chance that the computed difference of $(\approx 1.1 \mathrm{pK}$ units $)$ is in fact practically null. However, there is also a non-negligible chance that the $\mathrm{pKa}$ difference is even larger than $1.1 \mathrm{pK}$ units. Thus, we hope this result are enough to encourage experimentalists to perform the experimental design we propose. 
RESULTS AND DISCUSSION ANALYSIS OF THE PKA VARIATIONS AS A FUNCTION OF PH

Figure 2 shows a superposition of the lowest-energy conformations for both the native-like (green-ribbon) and the mirror-image (yellow-ribbon) of protein Q10H obtained by using ROSETTA Bradley et al. (2005). These two structures were used to compute for each ionizable residue along the sequence the value of the pKa variations $\left(\Delta=\left[p K_{\text {Native }}^{a}-p K_{\text {Mirror }}^{a}\right]\right)$ at $\mathrm{pH}$ 7.0 Vorobjev et al. (2008). The result of this analysis is shown in Figure 3 (as blue dots) where one of the largest change in $\Delta$, namely larger than $\pm 1.0 \mathrm{pK}$ units, occurs for H10. This large shift on the pKa of residue $\mathrm{H} 10$ appears to be a consequence of the close proximity of H10 to D38 in the mirror-image conformation of protein Q10H (see Figure 2).

There is another change of $\Delta$ larger than $\pm 1.0 \mathrm{pKa}$ unit and it occurs for residue K8 (see blue dots in Figure 3), a residue belonging to the flexible N-terminal region of the mutant protein Q10H, viz., ranging from residues $\mathrm{T} 1$ trough $\mathrm{E} 9$. The origin of the large computed shift in the $\mathrm{pKa}$ of residue $\mathrm{K} 8$ is the following. In the native structure of protein Q10H residue $\mathrm{K} 8$ is well exposed to the solvent. On the other hand, in the mirror image of $\mathrm{Q} 10 \mathrm{H}$ residue $\mathrm{K} 8$ is close to E16, making a favorable electrostatic interaction. However a close inspection of these two structures indicates that the favorable electrostaticinteraction between $\mathrm{K} 8$ and E16, observed in the mirror image conformation, could also occur on the native conformation, e.g., by a rearrangement of the backbone-torsional angles of the flexible $\mathrm{N}$-terminal region of the protein Q10H. If this were feasible, the computed pKa shift for K8 should be $\approx 0$. Consequently, monitoring the pKa shift of $\mathrm{K} 8$ does not appear to be the right choice for the purpose of an accurate determination of the coexistence between the native and the mirror image states in solution. Unlike the origin of the pKa shift for K8, the interaction between H10 and D38 cannot take place in both the native and the mirror-image conformations (see Figure 2) and, hence, from here on we will focus our attention on H10 only.

Consideration of the protein dynamics in water is very important for an accurate computation of conformational-dependent values, such as the pKa's. However, this effect was not taken into account in the computation of the $\Delta$ values shown, as blue-dots, in the Figure 3. Consequently, we carried out a constant-pH MD simulation of both the native-like Q10H mutant and its mirror-image conformations Vorobjev et al. (2018). As mentioned in the Materials and Methods section, during the simulations at constant-pH 7.0 it is reasonable to consider all acid (Asp, Glu) and base (Lys, Arg) residues in the ionized state, because their respective $\mathrm{pK}_{0}$ 's $(3.5,4.0$, and $10.5,12.5$, respectively) are shifted by more than 2.5 $\mathrm{pK}$ units from the $\mathrm{pH}$ (7.0) at which the calculations were carried out. For the same reason, the only Tyr in the sequence was consider as neutral. However, histidine residue pKa's (6.5) can vary considerably at $\mathrm{pH} 7.0$ at which the calculations are carried out and, hence, consideration of histidine ionization states for each of the imidazole ring of His forms must be considered explicitly. Consequently during the calculations the nine ionizations states of the two interacting His, namely between $\mathrm{H} 10$ and H19, were explicitly considered (see Table S2 of supplemental files). The average $\Delta$ change for H10, computed from the native-like and mirror-image conformations after $25 \mathrm{~ns}$ MD simulations, is shown as an orange dot in Figure 3. Similarly, the computed average change for the imidazole ring forms of $\mathrm{H} 10$ as a function of $\mathrm{pH}$ for both the native like and the mirror image conformations are display in Figure 4. As shown in this Figure at a given fix $\mathrm{pH}$, e.g., at $\mathrm{pH}=8.0$, there are significant changes among the computed fractions of the imidazole ring forms of $\mathrm{H} 10$.

In general, the results shown in Figures 3 and 4 and Table S3 (supplemental files) are decisive for the determination of the fraction of native and mirror image conformations in solution. Indeed, if the dominant conformation in solution is the native like then the $\mathrm{pKa}$ of $\mathrm{H} 10$ will be $6.2 \pm 0.2$. On the other hand, if the dominant conformation in solution is the mirror image then the pKa will be $7.3 \pm 0.2$. Any other in-between value may indicate coexistence of these two conformations in solution.

\section{Validation of the $\mathrm{H} 10$ pKa-based predictions}

Small changes around the computed average pKa value for H10 in the native-like conformation (6.2) are of course possible. In such a case additional experiments are necessary to determine whether such shift is due to expected fluctuations of the native conformation (around $\pm 0.3 \mathrm{in} \mathrm{pKa}$ units) or to the presence of a small fraction of the mirror-image conformation. One such additional experiment could be the determination of the tautomers of the imidazole ring of H10. In this section we analyze this possibility by using two NMR-based methods. 
First, as shown in Figures 4, there is a large change in the average fractions of H10 tautomers as a function of $\mathrm{pH}$. In particular, if the population of the native-like conformation is dominant in solution $(\approx 100 \%)$ then, as shown in Figure 4 , the fraction of the protonated form should be $\approx 0 \%$ at $\mathrm{pH} \approx 8.0$. In other words, only the imidazole ring of $\mathrm{H} 10$ tautomers will be present in solution at this $\mathrm{pH}$. Therefore, their relative populations can be determined accurately by measuring the one-bond $\mathrm{CH},{ }^{1} J_{C H}$, Spin-Spin Coupling Constants (SSCC) of the imidazole ring of H10.

Let us explain this in detail. Under the only condition that His is non-protonated, we have been able to show that the fraction of the $N^{\delta 1}-H$ tautomeric form $\left(f^{\delta 1}\right)$ of the imidazole ring of His can be estimated by using the following equation: $f^{\delta 1}=\left(J^{o b s}-165.0\right) / 15.0$, Vila and Scheraga (2017) where $J$ refers to ${ }^{1} J_{C \delta 2 H}$ SSCC, and here $o b s$ is the observed value in solution for H10. Naturally, $f^{\varepsilon 2}=1-f^{\delta 1}$. Hence, if the native-like structure is the dominant topology in solution, then the following inequality should hold: $f^{\varepsilon 2} \gg f^{\delta 1}$ (see Figure 4) otherwise there would be coexistence of the native-like structure with other topology in solution.

A second, and less restrictive, validation test will be to use a recently proposed NMR-based methodology aimed to determine the tautomeric forms as a function of the ionization state of the imidazole ring of histidine Vila et al. (2011). In this approach, the average tautomeric fraction of the $N^{\varepsilon 2}-H$ form of His $\left(f^{\varepsilon 2}\right)$ can be determined by using the following equation: $f^{\varepsilon 2}=\Delta^{o b s}\left(1-f^{H+}\right) / \Delta^{\varepsilon}$ where $f^{H+}$ is the experimentally determined fraction for the ionized form of $\mathrm{H} 10$, at a given fix $\mathrm{pH} ; \Delta^{o b s}=\left|{ }^{13} C^{\delta 2}-{ }^{13} C^{\gamma}\right|$, where ${ }^{13} \mathrm{C}^{\delta 2}$ and ${ }^{13} \mathrm{C}^{\gamma}$ are the NMR-observed chemical shifts for the imidazole ring of $\mathrm{H} 10$ at that $\mathrm{pH}$; and $\Delta^{\varepsilon}$ is the first-order absolute shielding difference, $\left|{ }^{13} C^{\delta 2}-{ }^{13} C^{\gamma}\right|{ }^{\varepsilon}$, between the ${ }^{13} C^{\delta 2}$ and ${ }^{13} C^{\gamma}$ nuclei for the $N^{\varepsilon 2}-H$ tautomer, i.e., present to the extent of $100 \%$. $\Delta^{\varepsilon}$ is a parameter which must be estimated Vila et al. (2011). As a first approximation, a $\Delta^{\varepsilon}=27.0 \mathrm{ppm}$, obtained from the analysis of a His-rich protein, Vorobjev et al. (2017) namely Loligo vulgaris (pdb id 1E1A), a 314-residue all- $\beta$ protein, Scharff et al. (2001) should be used. Naturally, the $f^{\delta 1}$ fraction, viz., for the $N^{\delta 1}-H$ tautomer, is obtained straightforwardly as: $f^{\delta 1}=1-f^{H+}-f^{\varepsilon 2}$. Although this second approach to compute the tautomers of $\mathrm{H} 10$ it is more general than the previous one, i.e., by using the ${ }^{1} J_{C \delta 2 H} \mathrm{SSCC}$, the determination of the ${ }^{13} \mathrm{C}^{\gamma}$ chemical shift it is not always feasible. Indeed, only $213{ }^{13} \mathrm{C}^{\gamma}$, versus $6,984{ }^{13} \mathrm{C}^{\delta 2}$, chemical shifts of the imidazole ring of histidine have been deposited in the Biological Magnetic Resonance data Bank (BMRB) Ulrich et al. (2008). Overall, if it were feasible to observe the ${ }^{13} \mathrm{C}^{\gamma}$ chemical shift we suggest to use both approaches to validate the pKa predictions.

Although this work is not intended to be a revision of all existing methods used to determine the tautomeric forms of the imidazole ring of His, the use of the tautomeric identification by direct observation of ${ }^{15} \mathrm{~N}$ chemical shifts of the imidazole ring of His, which is a common practice in NMR spectroscopy, Pelton et al. (1993); Shimahara et al. (2007); Hass et al. (2008) should be mentioned. This method requires, as a necessary condition, knowledge of the canonical limiting values of the ${ }^{15} \mathrm{~N}$ chemical shift of the imidazole ring of His in which each form of His is present to the extent of $100 \%$. In this regard, there is theoretical evidence indicating that a considerable difference for the average tautomeric equilibrium constant, $K_{T}$, can be obtained if DFT-computed ${ }^{15} \mathrm{~N}$ limiting values rather than canonical limiting values are used Vila (2012), Because these results raise concerns about the magnitude of the uncertainty associated with the predictions we did not consider this method as an alternative to the above-proposed tests to validate the pKa predictions.

All in all, the estimated tautomeric forms of the imidazole ring of His are certainly not enough to accurately determine whether the coexistence of native-like and mirror-image structures occurs in solution but it could be of valuable assistance to validate the determination made by the $\mathrm{pKa}$ analysis.

\section{CONCLUSIONS}

We provided a proof-of-concept of an experimental design that could be used to detect the coexistence of native and mirror-image conformations for the $\mathrm{Q} 10 \mathrm{H}$ mutant of protein $\mathrm{A}$ in solution. Determination of the pKa values of the ionizable residue H10 should provide a quick answer to this problem. Additionally the NMR-determination of the one-bond vicinal coupling constant or the chemical-shifts of the imidazole ring of $\mathrm{H} 10$ could be used to validate this finding. There are two main advantages of the proposed methodology. Firstly, there is no need for 3D structural information and, secondly, a validation test can be carried out by standard NMR-based experiments.

Whatever the output of the proposed experiments is, we will find them interesting. Indeed, if the results don't indicate the presence of the mirror image, all the theoretical predictions about the existence 
of the mirror image, published so far, would be only of Academic interest, perhaps, reduced only to show a possible intermediate conformational state in the pathway of protein folding. On the other hand, if the experiments provide evidence that there is structural coexistence, then the theoretical predictions will have a sound basis and, even more important, it may spur significant progress in the conformational analysis of proteins with mirror-images.

\section{ACKNOWLEDGEMENTS}

This research was supported by the Russian Foundation for Basic Research [15-04-00387-a], Russian Academy of Sciences [Program 'Molecular and Cell Biology, 6.11], MD modeling and pK calculations of a test set of proteins were supported by the Russian Science Foundation [16-14-10038] (YNV); U.S. National Institutes of Health [grant number GM-14312], U.S. National Science Foundation [grant number MCB10-19767] (HAS); CONICET-Argentina (PIP-0087], UNSL-Argentina (PROICO 3-2212), ANPCyT-Argentina (PICT-0556, PICT-0767) [JAV]

\section{ADDITIONAL INFORMATION}

The authors declare no competing interests.

\section{REFERENCES}

Bai, Y., Karimi, A., Dyson, H. J., and Wright, P. E. (1997). Absence of a stable intermediate on the folding pathway of protein A. Protein Science, 6(7):1449-1457.

Bermúdez, C., Mata, S., Cabezas, C., and Alonso, J. L. (2014). Tautomerism in Neutral Histidine. Angewandte Chemie - International Edition, 53(41):11015-11018.

Bradley, P., Chivian, D., Meiler, J., Misura, K. M. S., Rohl, C. A., Schief, W. R., Wedemeyer, W. J., Schueler-Furman, O., Murphy, P., Schonbrun, J., Strauss, C. E. M., and Baker, D. (2003). Rosetta Predictions in CASP5: Successes, Failures, and Prospects for Complete Automation. Proteins: Structure, Function and Genetics, 53(SUPPL. 6):457-468.

Bradley, P., Misura, K. M. S., and Baker, D. (2005). Toward High-Resolution de Novo Structure Prediction for Small Proteins. Science, 309(5742):1868-1871.

Chaudhury, S., Lyskov, S., and Gray, J. J. (2010). PyRosetta: A Script-Based Interface for Implementing Molecular Modeling Algorithms Using Rosetta. Bioinformatics, 26(5):689-691.

Deisenhofer, J. (1981). Crystallographic Refinement and Atomic Models of a Human Fc Fragment and Its Complex with Fragment B of Protein A from Staphylococcus aureus at 2.9- and 2.8-Å Resolution. Biochemistry, 20(9):2361-2370.

Dimitriadis, G., Drysdale, A., Myers, J. K., Arora, P., Radford, S. E., Oas, T. G., and Smith, D. A. (2004). Microsecond folding dynamics of the F13W G29A mutant of the B domain of staphylococcal protein A by laser-induced temperature jump. Proceedings of the National Academy of Sciences, 101(11):3809-3814.

Garcia, A. E. and Onuchic, J. N. (2003). Folding a protein in a computer: An atomic description of the folding/unfolding of protein A. Proceedings of the National Academy of Sciences, 100(24):1389813903.

Gouda, H., Torigoe, H., Arata, Y., Shimada, I., Saito, A., and Sato, M. (1992). Three-Dimensional Solution Structure of the B Domain of Staphylococcal Protein A: Comparisons of the Solution and Crystal Structures. Biochemistry, 31(40):9665-9672.

Hass, M. A. S., Hansen, D. F., Christensen, H. E. M., Led, J. J., and Kay, L. E. (2008). Characterization of conformational exchange of a histidine side chain: Protonation, rotamerization, and tautomerization of His61 in plastocyanin from Anabaena variabilis. Journal of the American Chemical Society, 130(26):8460-8470.

Kachlishvili, K., Maisuradze, G. G., Martin, O. A., Liwo, A., Vila, J. A., and Scheraga, H. A. (2014). Accounting for a mirror-image conformation as a subtle effect in protein folding. Proceedings of the National Academy of Sciences, 111(23):8458-8463.

Lazaridis, T. and Karplus, M. (1999). Effective energy function for proteins in solution. Proteins: Structure, Function, and Bioinformatics, 35(2):133-152. 
Lee, B. M., Xu, J., Clarkson, B. K., Martinez-Yamout, M. A., Dyson, H. J., Case, D. A., Gottesfeld, J. M., and Wright, P. E. (2006). Induced fit and "lock and key" recognition of 5 S RNA by zinc fingers of transcription factor IIIA. Journal of Molecular Biology, 357(1):275-291.

Machuqueiro, M. and Baptista, A. M. (2011). Is the prediction of pKa values by constant-pH molecular dynamics being hindered by inherited problems? Proteins: Structure, Function and Bioinformatics, 79(12):3437-3447.

Myers, J. K. and Oas, T. G. (2001). Preorganized secondary structure as an important determinant of fast protein folding. Nature Structural Biology, 8(6):552 - 558.

Noel, J. K., Schug, A., Verma, A., Wenzel, W., Garcia, A. E., and Onuchic, J. N. (2012). Mirror images as naturally competing conformations in protein folding. Journal of Physical Chemistry $B$, 116(23):6880-6888.

Olszewski, K. A., Kolinski, A., and Skolnick, J. (1996). Folding simulations and computer redesign of protein A three-helix bundle motifs. Proteins: Structure, Function and Genetics, 25(3):286-299.

Pelton, J. G., Torchia, D. A., Meadow, N. D., and Roseman, S. (1993). Tautomeric states of the active-site histidines of phosphorylated and unphosphorylated IIIGlc, a signal-transducing protein from escherichia coli, using two-dimensional heteronuclear NMR techniques. Protein Science, 2(4):543-558.

Popov, A. V. and Vorob'ev, Y. N. (2010). GUI-BioPASED: A program for molecular dynamics simulations of biopolymers with a graphical user interface. Molecular Biology, 44(4):648-654.

Sato, S., Religa, T. L., Daggett, V., and Fersht, A. R. (2004). From The Cover: Testing protein-folding simulations by experiment: B domain of protein A. Proceedings of the National Academy of Sciences, 101(18):6952-6956.

Scharff, E. I., Koepke, J., Fritzsch, G., Lücke, C., and Rüterjans, H. (2001). Crystal structure of diisopropylfluorophosphatase from Loligo vulgaris. Structure, 9(6):493-502.

Schnell, J. R. and Chou, J. J. (2008). Structure and mechanism of the M2 proton channel of influenza A virus. Nature, 451(7178):591-595.

Shimahara, H., Yoshida, T., Shibata, Y., Shimizu, M., Kyogoku, Y., Sakiyama, F., Nakazawa, T., Tate, S. I., Ohki, S. Y., Kato, T., Moriyama, H., Kishida, K. I., Tano, Y., Ohkubo, T., and Kobayashi, Y. (2007). Tautomerism of histidine 64 associated with proton transfer in catalysis of carbonic anhydrase. Journal of Biological Chemistry, 282(13):9646-9656.

Song, Y., Mao, J., and Gunner, M. R. (2009). MCCE2: Improving protein pKa calculations with extensive side chain rotamer sampling. Journal of Computational Chemistry, 30(14):2231-2247.

Ulrich, E. L., Akutsu, H., Doreleijers, J. F., Harano, Y., Ioannidis, Y. E., Lin, J., Livny, M., Mading, S., Maziuk, D., Miller, Z., Nakatani, E., Schulte, C. F., Tolmie, D. E., Kent Wenger, R., Yao, H., and Markley, J. L. (2008). BioMagResBank. Nucleic Acids Research, 36(SUPPL. 1):402-408.

Vila, J. A. (2012). Limiting Values of the Histidine at High pH. Journal of Physical Chemistry B, 116:6665-6669.

Vila, J. A., Arnautova, Y. A., Vorobjev, Y., and Scheraga, H. A. (2011). Assessing the fractions of tautomeric forms of the imidazole ring of histidine in proteins as a function of $\mathrm{pH}$. Proceedings of the National Academy of Sciences, 108(14):5602-5607.

Vila, J. A., Ripoll, D. R., and Scheraga, H. A. (2003). Atomically detailed folding simulation of the B domain of staphylococcal protein A from random structures. Proceedings of the National Academy of Sciences of the United States of America, 100(25):14812-14816.

Vila, J. A. and Scheraga, H. A. (2017). Limiting values of the one-bond C-H spin-spin coupling constants of the imidazole ring of histidine at high-pH. Journal of Molecular Structure, 1134:576-581.

Vorobjev, Y. N., Scheraga, H. A., and Vila, J. A. (2017). A comprehensive analysis of the computed tautomer fractions of the imidazole ring of histidines in Loligo vulgaris. Journal of Biomolecular Structure and Dynamics, 1102:1-12.

Vorobjev, Y. N., Scheraga, H. A., and Vila, J. A. (2018). Coupled molecular dynamics and continuum electrostatic method to compute the ionization $\mathrm{pKa}$ 's of proteins as a function of $\mathrm{pH}$. Test on a large set of proteins. Journal of Biomolecular Structure and Dynamics, 36(3):561-574.

Vorobjev, Y. N., Vila, J. A., and Scheraga, H. A. (2008). FAMBE-pH: A fast and accurate method to compute the total solvation free energies of proteins. Journal of Physical Chemistry B, 112(35):1112211136. 


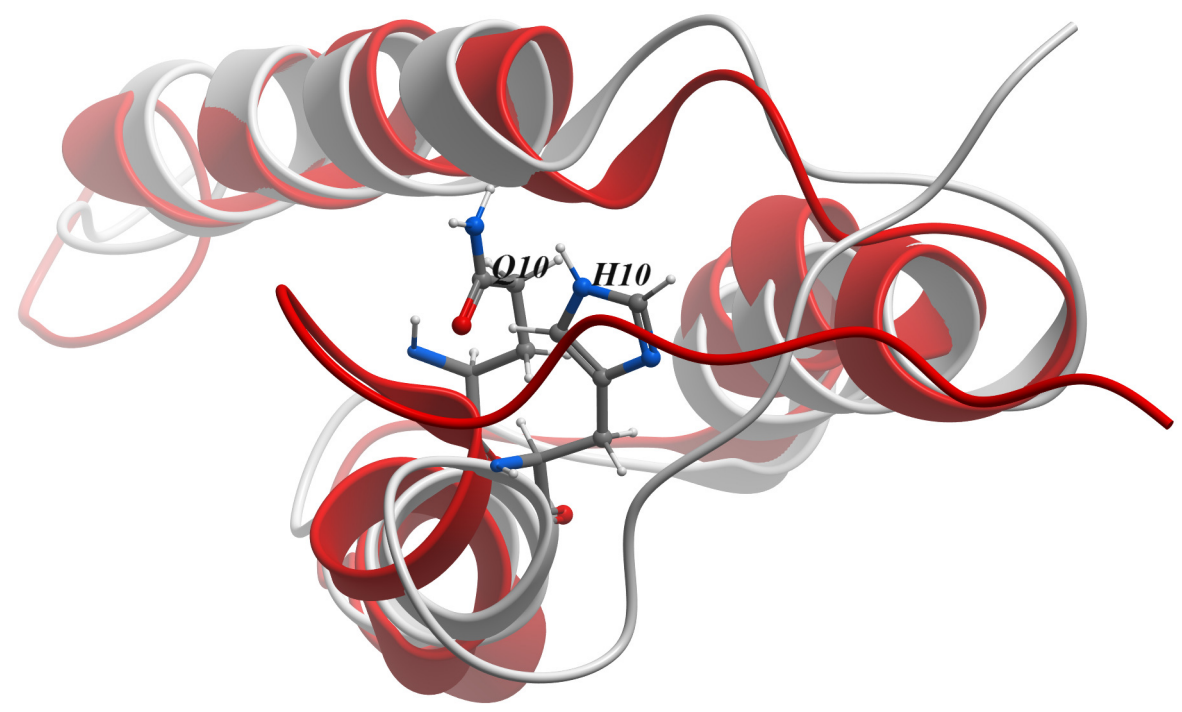

Figure 1. Red- and white-ribbon diagrams for the native structures of protein A (PDB ID 1BDD Gouda et al. (1992)) and the equivalent for protein Q10H, respectively. The position of the side-chain of Q10 and $\mathrm{H} 10$ for protein A and protein $\mathrm{Q} 10 \mathrm{H}$ are highlighted. The $C^{\alpha}$ rmsd between the two native structures is $1.4 \AA$.

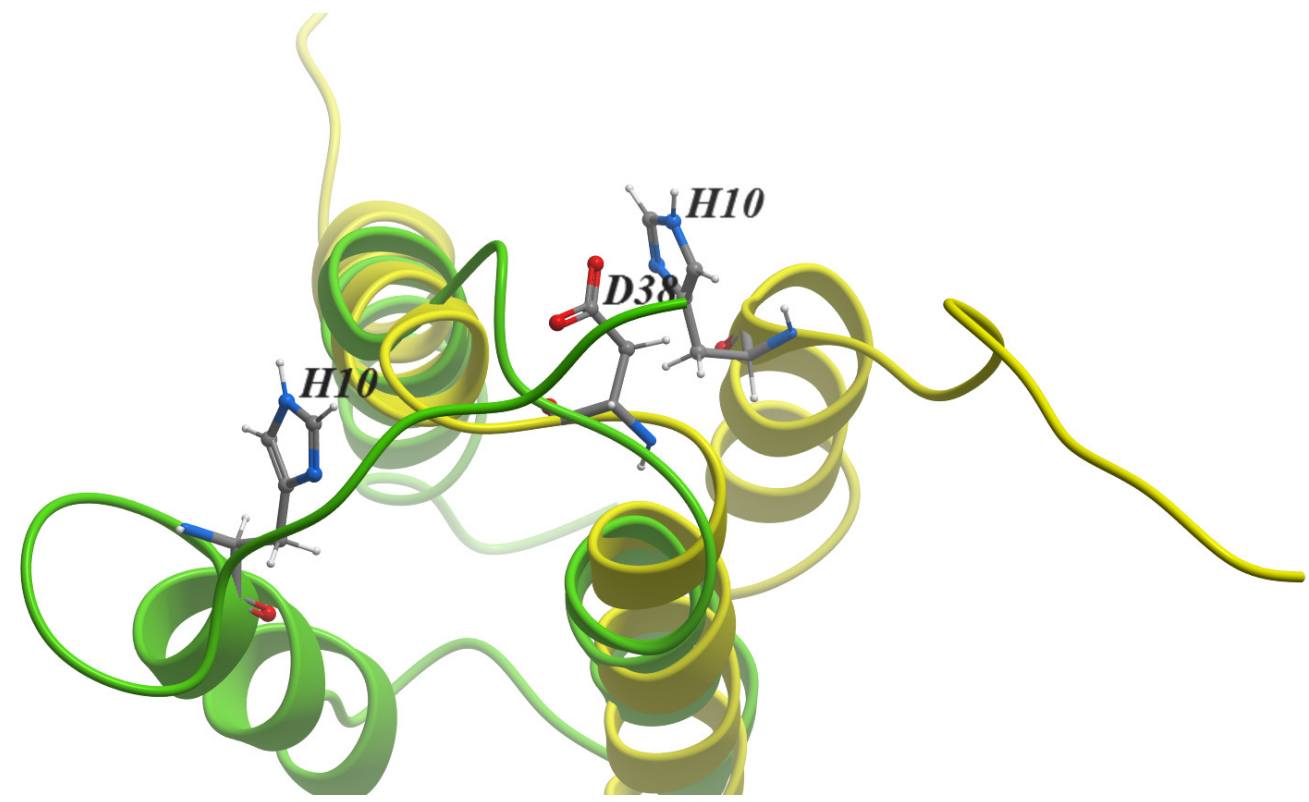

Figure 2. Green- and yellow-ribbon diagrams for the native and "mirror" image conformations of Protein A, respectively. The position of the side-chain of H10 is highlighted for each of these conformations. Moreover, the side-chain of D38 is also displayed to point out the close proximity between D38 and H10 in the "mirror" image conformation. The favorable electrostatic interaction between D38 and H10 may be responsible for the large $(\Delta \approx-1.1)$ change in the computed pKa between the native-like and the "mirror-image conformations. 


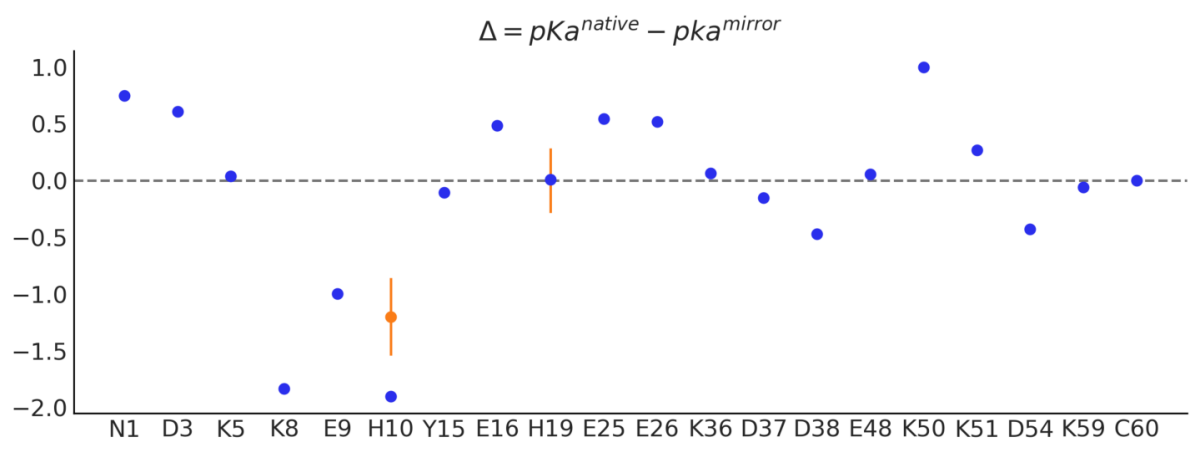

Figure 3. Dots indicate the $\mathrm{pKa}$ change $(\Delta)$, computed at $\mathrm{pH} 7.0$, for each ionizable residue along the protein $\mathrm{Q} 10 \mathrm{H}$ sequence. The blue-dots were computed from the single lowest-energy generated conformations of both the native-like and mirror-image topology, respectively. The orange-dots were computed for the two histidines in the sequence, namely H10 and H19, as an average over 25ns MD simulations for both the native-like and mirror-image conformations; vertical orange-lines denotes the standard deviations of the computed average $\Delta$ values 


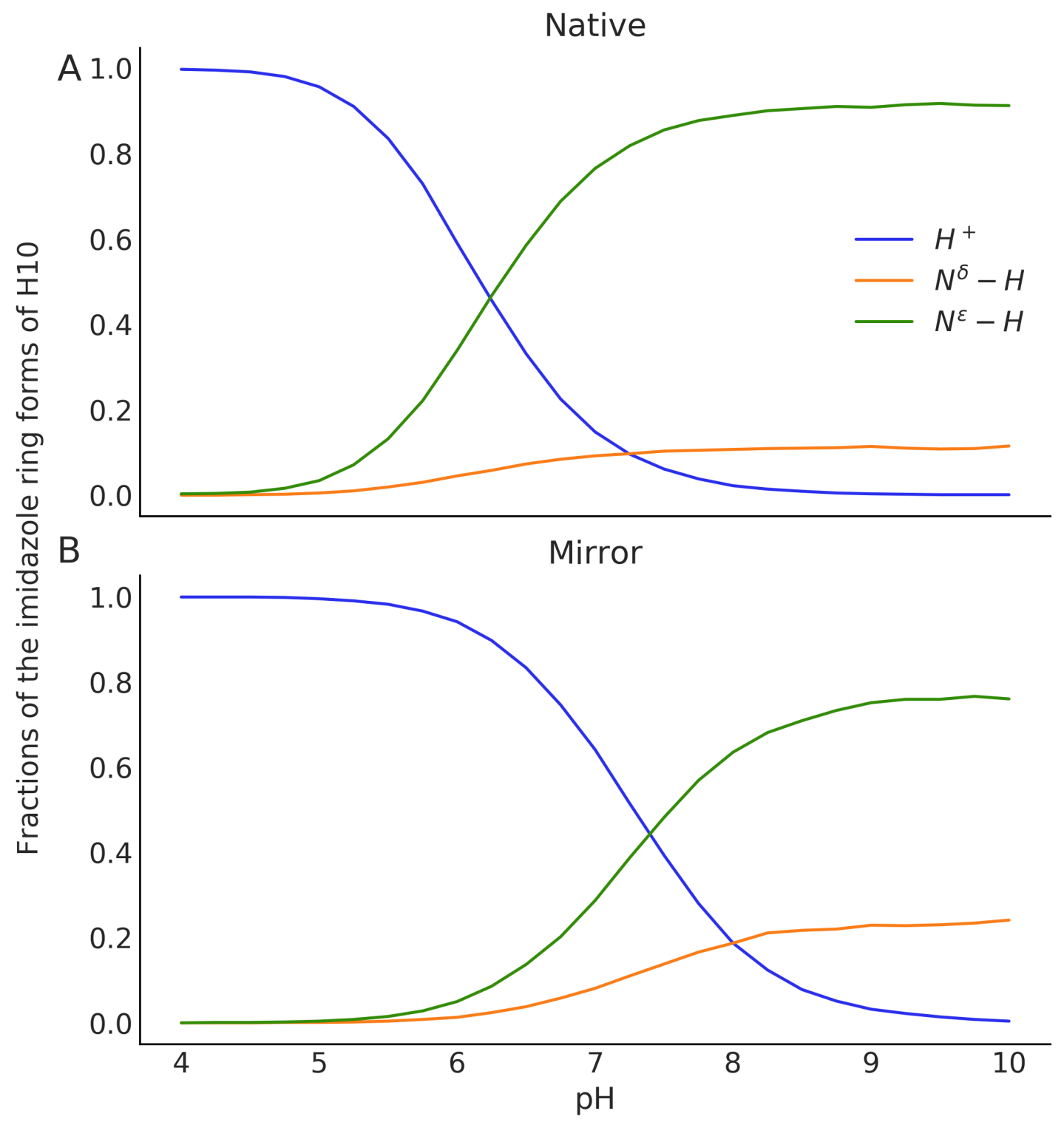

Figure 4. Fractions of the imidazol ring forms of $\mathrm{H} 10$ as a function of $\mathrm{pH}$, for the "Native" (panel A) and "Mirror" (panel B) topologies of the Q10H mutant of protein A. The values, for each topology, are estimated along 25ns MD equilibrium trajectory for each of nine ionization states of two electrostatically-coupled histidines residues, namely H10 and H19 